\title{
Design and development of a profilometer for the fast and accurate characterization of optical surfaces
}

\author{
José A. Gómez-Pedrero*a, Diego Rodríguez-Ibañez ${ }^{\mathrm{b}}$, José Alonso ${ }^{\mathrm{a}, \mathrm{b}}$, Juan A. Quiroga ${ }^{\mathrm{a}, \mathrm{c}}$ \\ ${ }^{a}$ Applied Optics Complutense Group, Universidad Complutense de Madrid, Faculty of Optics and \\ Optometry, Avda. Arcos de Jalón 118, 28037, Madrid, SPAIN; \\ ${ }^{\mathrm{b}}$ Indizen Optical Technolgies, C. Santa Engracia 6, 28011, Madrid, SPAIN; \\ ${ }^{c}$ Applied Optics Complutense Group, Universidad Complutense de Madrid, Faculty of Physics, \\ Ciudad Universitaria s/n, 28040, Madrid, SPAIN.
}

\begin{abstract}
With the advent of techniques devised for the mass production of optical components made with surfaces of arbitrary form (also known as free form surfaces) in the last years, a parallel development of measuring systems adapted for these new kind of surfaces constitutes a real necessity for the industry. Profilometry is one of the preferred methods for the assessment of the quality of a surface, and is widely employed in the optical fabrication industry for the quality control of its products. In this work, we present the design, development and assembly of a new profilometer with five axis of movement, specifically suited to the measurement of medium size (up to $150 \mathrm{~mm}$ of diameter) "free-form" optical surfaces with sub-micrometer accuracy and low measuring times. The apparatus is formed by three $\mathrm{X}, \mathrm{Y}, \mathrm{Z}$ linear motorized positioners plus and additional angular and a tilt positioner employed to locate accurately the surface to be measured and the probe which can be a mechanical or an optical one, being optical one a confocal sensor based on chromatic aberration. Both optical and mechanical probes guarantee an accuracy lower than the micrometer in the determination of the surface height, thus ensuring an accuracy in the surface curvatures of the order of $0.01 \mathrm{D}$ or better. An original calibration procedure based on the measurement of a precision sphere has been developed in order to correct the perpendicularity error between the axes of the linear positioners. To reduce the measuring time of the profilometer, a custom electronics, based on an Arduino ${ }^{\mathrm{TM}}$ controller, have been designed and produced in order to synchronize the five motorized positioners and the optical and mechanical probes so that a medium size surface (around $10 \mathrm{~cm}$ of diameter) with a dynamic range in curvatures of around $10 \mathrm{D}$, can be measured in less than 300 seconds (using three axes) keeping the resolution in height and curvature in the figures mentioned above.
\end{abstract}

Keywords: Profilometry, optical surfaces, accuracy, free-form optics

\section{INTRODUCTION}

The term "Free-form Optics" refers to those optical systems which present, at least, one surface of arbitrary form, usually highly aspherical and non-symmetrical, quite different to the spherical or symmetrical aspherical surfaces traditionally employed in Optics. Examples of these systems can be found for both imaging and non-imaging optics. For the first group, free-form surfaces are widely employed nowadays in the manufacturing of ophthalmic lenses, particularly progressive addition ones [1][2]. Free-form imaging optics is also present in the design of enhanced imaging systems ranging from high aspect on-axis systems [3] to both optical [4] and catoptrical [5] photographic lenses. The main applications of non-imaging optical systems with free form surfaces are illumination systems [6] and solar concentrators [7]. The usage of free-form surfaces allows for a greater number of degrees of freedom for the optical designer enabling, in this way, the design of enhanced optical systems.

An important topic in the field of Free-form Optics is the manufacturing and testing of the free-form surfaces. In this context, measuring free-form surfaces is particularly challenging as is the case of progressive lens surfaces. Progressive surfaces present great apertures, ranging from 60 to $90 \mathrm{~cm}$ of diameter, an elevation range of several millimeters, and curvature variations of several diopters along the surface [1]. Moreover, the usual practice of the ophthalmic lens manufacturing industry is to design a different surface for each prescription (refractive error plus value of addition) and material which means that the number of surfaces to be measured can be estimated in the hundreds. The accuracy requirements are also tight: around 1 micron for the elevation and $0.01 \mathrm{D}$ for the local curvature of the surface. In these

Optical Systems Design 2015: Optical Fabrication, Testing, and Metrology V, edited by Angela Duparré, Roland Geyl, Proc. of SPIE Vol. 9628, 96281I

(C) 2015 SPIE · CCC code: 0277-786X/15/\$18 - doi: 10.1117/12.2191058 
conditions, the assessment of the quality of a progressive surface is generally carried out by using a coordinate measuring machine $(\mathrm{CMM})$ also known as a profilometer.

The advantages of profilometry are the accuracy in both horizontal positioning (which corresponds to the coordinates $\mathrm{X}$ and $\mathrm{Y}$ ) and in the measurement of the elevation (coordinate Z). Using motorized positioners driven by step motors, the accuracy in the horizontal position of the sample can be as low as 0.1 microns, while state-of-the-art mechanical probes can yield accuracies of the order of 50 nanometers which are enough to meet the requirements of accuracy for ophthalmic surfaces stated in the preceding paragraph. However, standard CMM devices also present some drawbacks being the foremost of them low measuring times and the possibility of causing permanent damage to the measured surface due to mechanical friction between the sample surface and the tip of the probe.

One way to overcome the drawbacks of conventional profilometry is the substitution of the mechanical probe by an optical one avoiding, in this way, any damage on the lens surface while lowering simultaneously the measuring times due to the inherent speed of the optical measuring techniques. Indeed, in the last years a number optical probes based on different technologies have been commercialized. Among these technologies we found laser triangulation, reflectance measurement, confocal microscopy and chromatic confocal microscopy. Despite advantages such as non damaging measurement and speed, optical probes present a common drawback as the maximum slope of the surface that can be measured using those probes is limited and it is also considerably lower than maximum slope which can be measured by a contact tip.

In this work we present the design and development of a CMM for measuring free form surfaces, particularly progressive lens surfaces which present two differences when compared to conventional profilometers: 1) a dual mechanical/optical probe for measuring the surface elevation and 2) a motorized tilt and rotator units whose combined movement allows for the orientation of the measured surface in relation with the probe in such a way that the slopes of the surface are kept bellow the range of slopes that can be measured by the optical probe. The paper is organized as follows: in the next section we state the requirements of a CMM for the measurement of free form surfaces, with a special focus on the measurement of ophthalmic lens surfaces, then we will discuss the mechanical and electronic design of the device, afterwards, we will present the calibration procedure for a three-axes CCM and, finally, we will show some preliminary results obtained for the device operating in three-axes for a spherical and progressive addition lens surfaces.

\section{ACCURACY REQUIREMENTS FOR MEASURING FREE-FORM OPHTHALMIC SURFACES}

The most important parameter in order to establish the accuracy requirements for measuring free-form ophthalmic surfaces are the principal curvatures as they are related to the local power of the lens. Although in the general case, a simple analytical formulation of the local power in terms of surface curvature cannot be obtained, it is possible to get such simple formulation within the approximations stated in reference [8]. In this later case, the local curvatures of a surface $\kappa_{m}(x, y)$ and $\kappa_{M}(x, y)$ with $\kappa_{m}(x, y)<\kappa_{M}(x, y)$ are related to the local spherical and cylindrical refractive powers through the following relationship

$$
\begin{aligned}
& E(x, y)=\left(n^{\prime}-n\right) \cdot \kappa_{m}(x, y), \\
& C(x, y)=\left(n^{\prime}-n\right) \cdot\left(\kappa_{M}(x, y)-\kappa_{m}(x, y)\right),
\end{aligned}
$$

where $n$ and $n$ ' are the refractive index before and after the surface, respectively. Let us consider now a point of the surface given by the coordinates $\left(x_{0}, y_{0}, z_{0}\right)$. Then, in a neighborhood centered at this point and with a proper choice of the coordinate system, we can describe the surface as follows

$$
z(x, y) \cong \frac{1}{2} \kappa_{m} x^{2}+\frac{1}{2} \kappa_{M} y^{2},
$$

being $\kappa_{m}$ and $\kappa_{M}$, the local curvatures evaluated at the point $\left(x_{0}, y_{0}, z_{0}\right)$. If we consider now a spherical surface for which $\kappa \equiv \kappa_{m}=\kappa_{M}$, then within the limits where the approximation given by equation (2) holds, the local curvature can be written as

$$
\kappa \cong \frac{2 z}{x^{2}+y^{2}}=\frac{2 z}{r^{2}}
$$


being $r^{2}=x^{2}+y^{2}$. After applying the error propagation formula to equation (4), we can write the error in the curvature $\delta \kappa$ as

$$
\delta \kappa \cong\left\|\frac{2}{r^{2}}\right\| \delta z+\left\|\frac{4 z}{r^{3}}\right\| \delta r
$$

In ophthalmic optics, the usual figure for curvature accuracy is $0.01 \mathrm{~m}^{-1}$ (or 0.01 diopters). In order to make a rough estimation of the accuracy required for $z$ and $r$ to achieve this accuracy in the local curvature, let us consider a surface with $\kappa=15 \mathrm{~m}^{-1}$. In a point placed over this surface at a distance $r=0.01 \mathrm{~m}$ from the origin of coordinates, we have that, according to equation (3), the value of the elevation at this point should be $z=7.5 \times 10^{-4} \mathrm{~m}$. Substituting these figures in equation (4) we found that

$$
\delta \kappa\left(m^{-1}\right) \cong 2 \times 10^{4} \cdot \delta z(m)+3 \times 10^{3} \delta r(m),
$$

If we impose that each term of the right side of equation (5) be lower than the requested curvature accuracy, we found the following conditions:

$$
\begin{aligned}
& \delta z(m)<5 \times 10^{-7} \mathrm{~m}, \\
& \delta r(m)<3.33 \times 10^{-6} \mathrm{~m} .
\end{aligned}
$$

Therefore, we need a resolution of around 1 micron in the three axis of the CMM in order to measure curvatures of $15 \mathrm{D}$ with an accuracy of 0.01 diopters. However, accuracy is not the only requirement for performing a proper measurement of ophthalmic optics surfaces, as there are other constrains to be taken into account such as the surface diameter, range of surface curvatures, etc. Thus, we have summarized in Table 1 the specifications of our CMM for measuring ophthalmic lenses

Table 1. Summary of the specifications of the proposed profilometer.

\begin{tabular}{|ll|}
\hline Measurement length: & \\
Horizontal $(\mathrm{X}, \mathrm{Y})$ directions: $10 \mathrm{~cm}$ & Vertical (Z) direction: $5 \mathrm{~cm}$ \\
Accuracy: & 1 micron in the $\mathrm{X}, \mathrm{Y}, \mathrm{Z}$ direction \\
Resolution: & 0.1 micron in the $\mathrm{X}, \mathrm{Y}, \mathrm{Z}$ direction \\
Measuring speed: & Less than 300 seconds \\
Maximum surface curvature: & $15 \mathrm{D}$ \\
Curvature accuracy: & $0.01 \mathrm{D}$ \\
\hline
\end{tabular}

In the next section we will discuss the mechanical and electronic design developed in order to meet these specifications.

\section{MECHANICAL AND ELECTRONIC DESIGN OF THE PROFILOMETER}

\section{Mechanical design}

The goal of the mechanical design of the CMM profilometer is the correct positioning of the three basic elements which composed such system: the displacement stages, the measuring probe and the sample holder. The profilometer proposed has five motorized displacement units: three linear ones for the $\mathrm{X}, \mathrm{Y}$ and $\mathrm{Z}$ axis together with two additional tilt and rotation units. Those stages are commercial units manufactured by Newport and all the linear stages (Newport model GTS150) have a travel range of $150 \mathrm{~mm}$ with a positional accuracy of 1 microns, resolution of $0.05 \mu \mathrm{m}$ and a maximum travel speed of $50 \mathrm{~mm} / \mathrm{s}$. These specifications meet or even exceed in some cases the requirements compiled in table 1 . The two remaining angular displacement units (a tilt and a rotator) will be employed in conjunction with the optical probe to change the orientation of the lens surface so to avoid loss of light due to high local slopes (this feature will be discussed later). The motorized displacement stages are controlled by a commercial controller manufactured by Newport (model XPS-Q6 with capacity to control six axis) supplement by a custom made electronic module designed for a proper 
synchronization between the motorized stages and the measuring probes (optical and mechanical). We will give later a brief summary of the characteristics of this electronic module.

The motorized units are assembled together over an optical table using a set of custom designed mechanical elements. The design of these elements have been made using a 3D CAD software in which we have integrated the CAD designs of the commercial elements in order to ensure the proper assembly of all the components of the system. In figure 1a) we can see, as an example, the CAD of a preliminary version of the profilometer, showing the disposition of the motorized stages and its configuration as column profilometer. In figure $1 \mathrm{~b}$ ) we show the definitive configuration of the five-axis CMM. As it is shown in this photograph, the $\mathrm{X}$ and $\mathrm{Y}$ linear positioners are assembled together and above them we found, in ascending order from the linear stages, the tilt unit, the rotator and finally the sample holder. The $\mathrm{Z}$ translator together with the two optical and mechanical probes are assembled on a vertical structure positioned over the lens holder (figure 1b). Therefore, the lens surface will be moved in the $\mathrm{X}$ and $\mathrm{Y}$ directions (and tilted/rotated when necessary) for measuring the surface and the probes can be moved in the $\mathrm{Z}$ direction for an accurate positioning of the probe with respect to the lens surface before the actual measurement starts. Details of the assembly of those elements can be seen in figure 1c).

a)

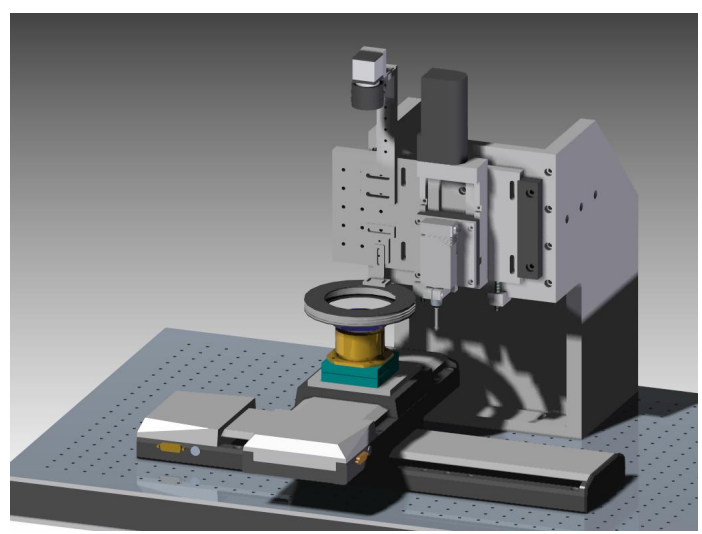

c)

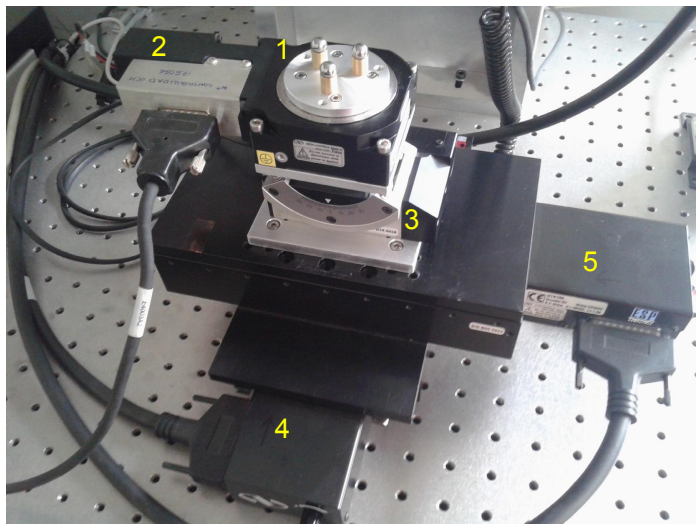

b)

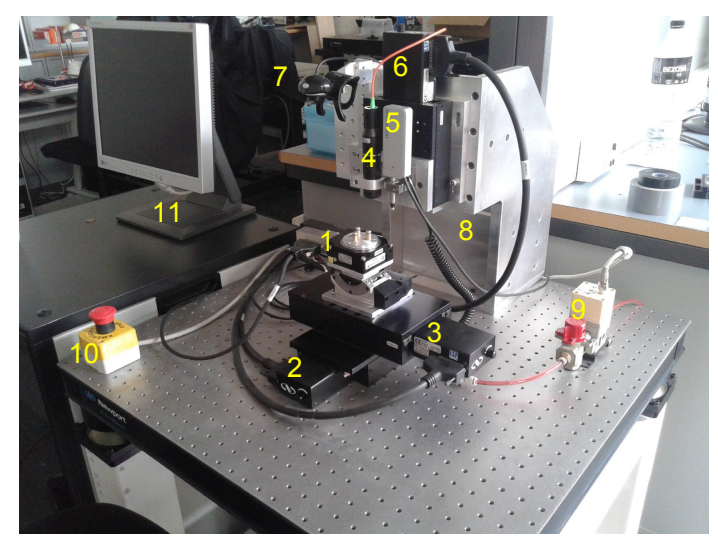

d)

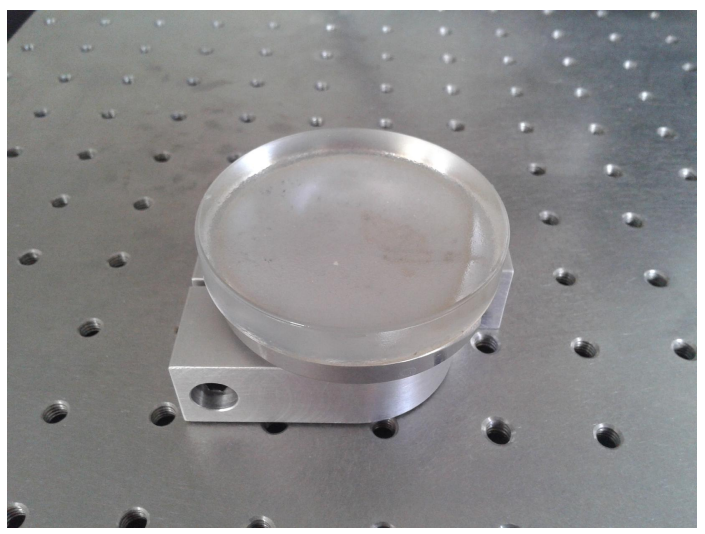

Figure 1. a) Preliminary CAD design of the profilometer with three axis, b) photograph of the definitive assembly of the five axis profilometer with the following elements 1 lens holder and tilt and rotation stages, 2 Y axis motorized stage, 3 ) $\mathrm{X}$ axis motorized stage, 4 optical probe, 5 mechanical probe, $6 \mathrm{Z}$ axis motorized stage, 7 CCD camera, 8 column support,

9 pneumatic regulator for the mechanical probe, 10 STOP button and 11 rack containing the electronic control and computer, c) detail of the profiometer 1 three point lens holder, 2 motorized rotator, 3 motorized tilt stage, 4 Y axis motorized stage and $5 \mathrm{X}$ axis motorized stage, d) alternative lens holder for blocked lenses.

The last element of the mechanical design is the lens holder. As we are dealing with ophthalmic lenses we have designed two holders specifically suited to this kind of lenses. The first one is a three point holder for finished lenses (see figure 
1c) for which the lens is fixed over three balls so any surface will be properly fixed with independence of its form. Thus, we can hold tightly even high aspherical surfaces such progressive ones. The other holder is designed for semifinished lenses which are still in the manufacturing process and, therefore, are blocked to a piece (named blocker) which ensures a secure holding of the lens. A photograph of this alternative holder is shown in figure 1d). A final element of the mechanical design is a mechanical arm for positioning a CCD camera which is employed in delimiting the bounds of the measuring area over the lens surface.

\section{Electronic design}

To describe the electronic design of the device it is important to start by describing the optical and mechanical probes employed. The usage of two probes gives more flexibility to the system which can measure hard surfaces with the mechanical probe and soft surfaces (such as the surfaces of lenses manufactured in plastic) with the optical one avoiding in this way any damage to the surface. The optical probe is faster than the mechanical one but the range of available slopes which can be measured with this device is limited by its numerical aperture. This is why we have included the rotator and tilt motorized stages in order to change the orientation and, consequently, the slope of the surface to be measured. The mechanical probe selected is the model MT 2587 manufactured by Heidenheim [9]. This sensor has a pneumatic regulator so the tip could extent a uniform pressure over the surface to be measured and it has an accuracy of 0.2 microns. The optical probe is the confocal sensor CL2-MG210 manufactured by Stil Optics [10] which is based on the combination of the confocal microscopy principle with the effect of chromatic aberration in order to measure accurately (up to $80 \mathrm{~nm}$ ) the surface elevation, although the range of slopes that can be measured is limited to $\pm 28^{\circ}$ due to the limitation imposed by the numerical aperture of the probe, as it has been told before.

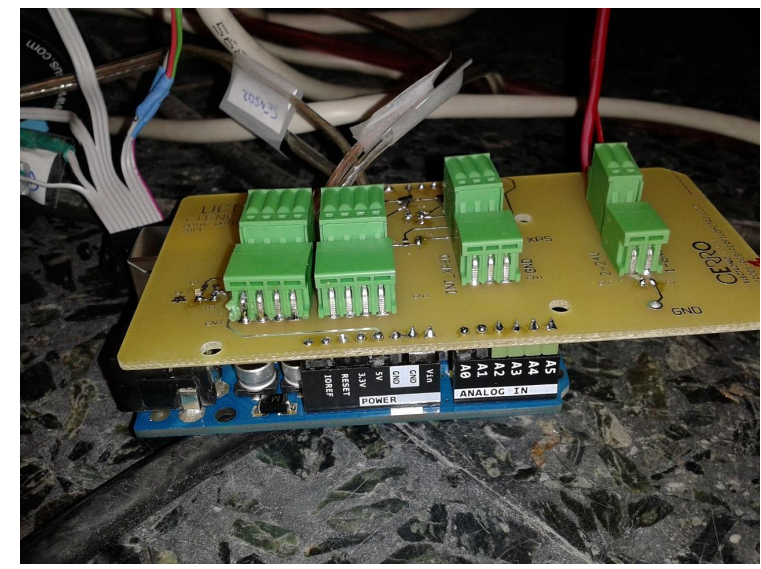

Figure 2. Photograph of the custom electronic controller for signal conditioning and triggering composed by a custom made interface board (upside) and an Arduino ${ }^{\mathrm{TM}}$ unit (downside).

In order to reduce the measuring time to the required levels, it is thus necessary to synchronize five motorized stages plus two probes (although only one of this probes will actually measure the surface at a time). The best way to achieve this goal is to employ the trigger signal supplied by the electronic controller of the motorized stages. Therefore, the motorized stages will move and, once they pass through a measuring point the electronic controller will send a trigger signal to the probe which, in turn, will measure the elevation of the surface at this instant. The problem with this scheme is that the signal trigger provided by the electronic controller is different in shape, duration and peak voltage to the input signal accepted by both sensors. Therefore a signal conditioning is required in order to perform a correct synchronous measurement. This conditioning is achieved using an Arduino ${ }^{\mathrm{TM}}$ controller in the way we will describe in the following paragraph.

Arduino $^{\mathrm{TM}}$ is an open-source platform based on a microcontroller and a SDK software designed for its implementation in applications such as signal processing and instrument control [11]. In our case, we have employed the unit Arduino UNO together with the protection box for Arduino ${ }^{\mathrm{TM}}$. The task of the unit is the detection of the trigger signal sent by the electronic controller of the motorized stages and, after its conditioning, the sending of this signal to the optical/mechanical sensor without any synchrony loss. To do this, a custom made interface board (see figure 2) has been 
designed and produced in order to connect the input signal coming from the electronic controller with the digital inputs of the Arduino ${ }^{\mathrm{TM}}$ unit. This signal will be processed by the Arduino ${ }^{\mathrm{TM}}$ which, in turn, will generate the correct output trigger for the optical/mechanical sensor. The electronic design of the system has been complemented by the installation of an STOP button and by the implementation of an electronic control for the pneumatic regulator of the mechanical probe.

\section{CALIBRATION OF THE $X$, Y AND Z AXES PERPENDICULARITY}

The CMM implemented is a Cartesian or column type device formed by a horizontal platform for displacing the sample in the $\mathrm{X}$ and $\mathrm{Y}$ direction and a vertical column for supporting the optical/mechanical probe which can be displaced along the $\mathrm{Z}$ axis. This kind of configuration is prone to present the so called squareness error which is particularly important for custom made CMM devices [12] as it is difficult to ensure the proper alignment of the three motorized stages with the required accuracy. In order to overcome this problem, we have employed the technique described in reference [12] due to its simplicity and easiness of implementation. We will describe now briefly the calibration procedure followed in order to remove the squareness error.

The relationship between the coordinates of given point measured in an purely Cartesian system $(x, y, z)$ and those measured in an Affine system $(u, v, w)$ which shares a common origin of coordinates with the former one is [12]

$$
\left[\begin{array}{l}
x \\
y \\
z
\end{array}\right]=\left[\begin{array}{ccc}
\cos \left(\delta_{U W}\right) & \sin \left(\delta_{V U}\right) \cos \left(\delta_{V W}\right) & 0 \\
0 & \cos \left(\delta_{V U}\right) \cos \left(\delta_{V W}\right) & 0 \\
\sin \left(\delta_{U W}\right) & \sin \left(\delta_{V W}\right) & 1
\end{array}\right] \cdot\left[\begin{array}{c}
u \\
v \\
w
\end{array}\right]
$$

Where $\delta_{\mathrm{UW}}, \delta_{\mathrm{VU}}$ and $\delta_{\mathrm{VW}}$ are the angles that forms each one of the three axis with the corresponding Cartesian axis, thus, for example, $\delta_{\mathrm{UW}}$ would be the angle formed by the $\mathrm{U}$ and $\mathrm{X}$ axis. Obviously, in a squareness free system, all of these angles would be zero so these angles stands for the squareness error. To compute these angles, the first step is to perform the measurement of a reference sphere. For a spherical surface of radius $R$ the following relationship holds between the coordinates $(x, y, z)$, referred to a perfect Cartesian system, of any point located over this surface:

$$
z=S(x, y) \equiv R \cdot\left(1-\sqrt{\left(1-\frac{\left(\left(x-x_{V}\right)^{2}+\left(y-y_{V}\right)^{2}\right)}{R^{2}}\right)}\right)
$$

by substituting the coordinates defined in (7) in equation (8) we arrive to the following expression [12]:

$$
w \equiv f(u, v)=-\sin \left(\delta_{U W}\right) u-\sin \left(\delta_{V W}\right) v-S\left(\cos \left(\delta_{U W}\right) u+\sin \left(\delta_{V U}\right) \cos \left(\delta_{V W}\right) v, \cos \left(\delta_{V U}\right) \cos \left(\delta_{V W}\right) v\right)
$$

In these conditions if we measure a set of $N$ calibration points $\left(u_{i}, v_{i}, w_{i}\right)_{i=1,2 \ldots N}$ we can define the following merit function

$$
\phi(R, V, \delta)=\sum_{i=1}^{N}\left(w_{i}-f\left(u_{i}, v_{i}\right)\right)^{2}
$$

Where $V$ stands for the coordinates $\left(x_{V}, y_{V}\right)$ and $\delta$ for the three angles $\delta_{\mathrm{UW}}, \delta_{\mathrm{VU}}$ and $\delta_{\mathrm{Vw}}$ which characterize the squareness error. Therefore, by solving equation (10) we will found the optimum values for the curvature radius of the sphere, the horizontal coordinates of the sphere vertex $\left(x_{V}, y_{V}\right)$ and the values of the angles related to the squareness error. As stated in reference [12] using this technique with a spherical surface of $\lambda / 8$ surface quality, calibration errors of around $10^{-3} \mathrm{rad}$ can be detected and corrected. This technique can be easily extrapolated to our five axes CMM in order to take into account the alignment errors of the rotator and tilt stages with respect to the Cartesian system. 


\section{PRELIMINARY RESULTS WITH THREE AXIS CONFIGURATION}

As an example of the capabilities of the CMM developed, we show the results obtained when measuring a spherical surface and then a progressive surface, the latter as an example of a free-form surface. In figure 3 a) we show the cloud of points belonging to a spherical surface measured by the CMM operating on three axis with the mechanical probe. We show also in this figure the path followed by the probe tip over the surface which is a minimum length one. After performing the fitting described in the previous section we obtained the residuals plotted in figure $3 \mathrm{~b}$ ). The goodness of the fit is indicated by the histogram of residues depicted in figure 3c) which shows a shape close to a Gaussian. Finally in figure $4 \mathrm{~b}$ ) we show the refractive power of the surface computed form the surface data supposing a refractive index of $n=1.5$ and using an exact ray tracing algorithm. Note that this refractive power is not constant over the surface due to the presence of aberrations, particularly oblique astigmatism and field curvature.

a)

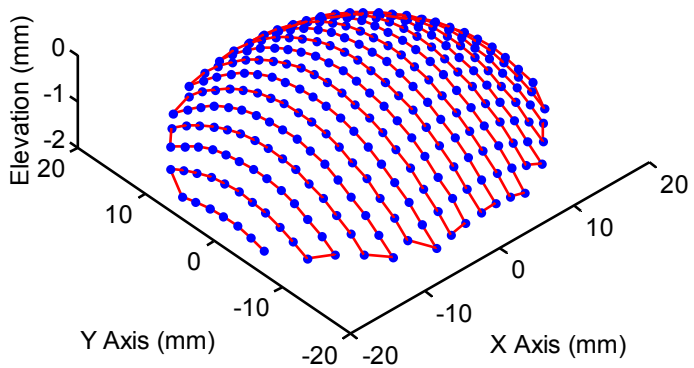

c)

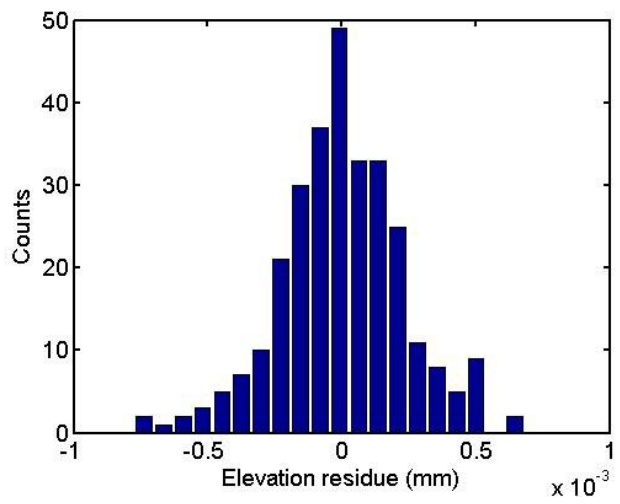

b)

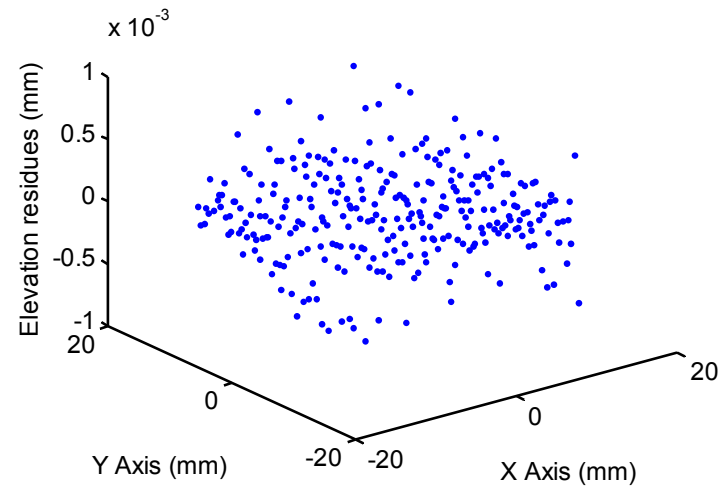

d)

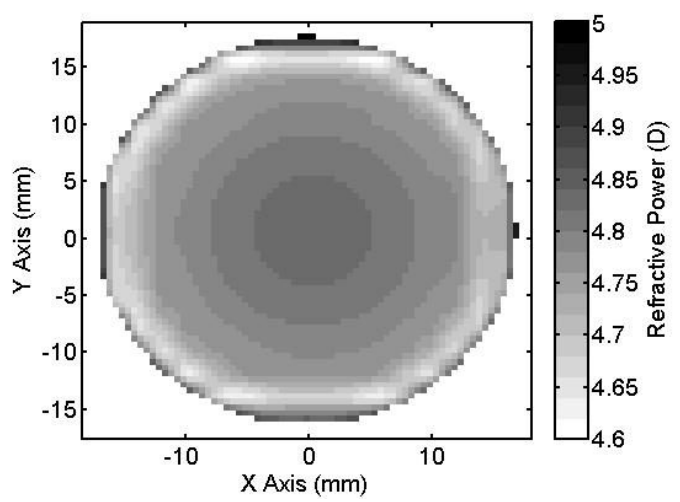

Figure 3. a) Plot of the points measured over a spherical surface using our CMM (blue dots) and minimum length path followed by the probe over the surface, $b$ ) residues obtained after fitting the cloud of points measured to a sphere $c$ ) histogram of the residues plotted in figure $4 \mathrm{~b}$ ) and d) map of spherical refractive power over the lens surface.

After fitting the measured points to a sphere using the calibration procedure described in the previous section, we found a maximum (peak-valley) error of $7.7 \times 10^{-4} \mathrm{~mm}$ being the radius of the sphere $105.8 \mathrm{~mm}$ (curvature of $9.45 \mathrm{D}$ ). As it is shown in figure $3 b$ ) the histogram of the residues of the fitting presents a shape similar to a Gaussian being the mean value for the error $8.4 \times 10^{-11} \mathrm{~mm}$ and the standard deviation $2.3 \times 10^{-4} \mathrm{~mm}$.

Finally, in figure 4 we show the refractive power maps (sphere and cylindrical power) computed from the surface data corresponding to a progressive surface measured by the CMM in three axis configuration with the mechanical probe. As it can be appreciated in these plots, the highly aspherical form of the surface leads to the progressive variation of 
spherical power which can be appreciated in figure 4a) and to the progressive corridor and the lobes of unwanted astigmatism shown in figure $4 \mathrm{~b}$ ). It is worth to notice that, although these results have been obtained using the mechanical probe, similar results would have been obtained if we had used the optical probe instead of the mechanical one. However, at this stage of the development of the profilometer we have not yet finished the development and programming of the algorithms for measuring surfaces with high slopes. Once this algorithm had been finished and tested, we will be able to measure surfaces with curvature ranging up to $15 \mathrm{D}$ using the optical probe with the consequent improvement in the measurement times.

a)

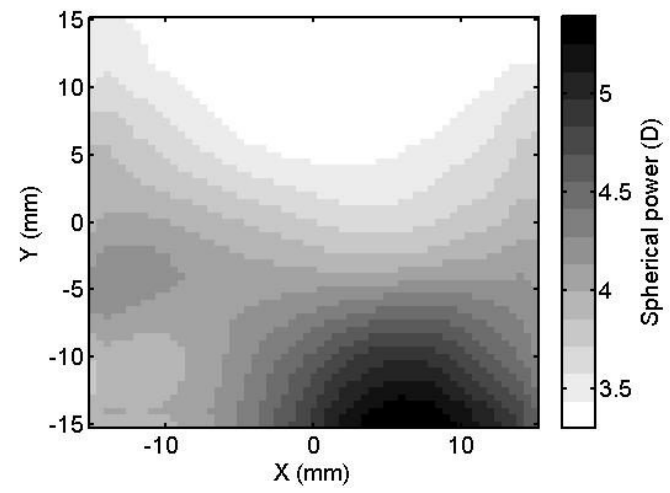

b)

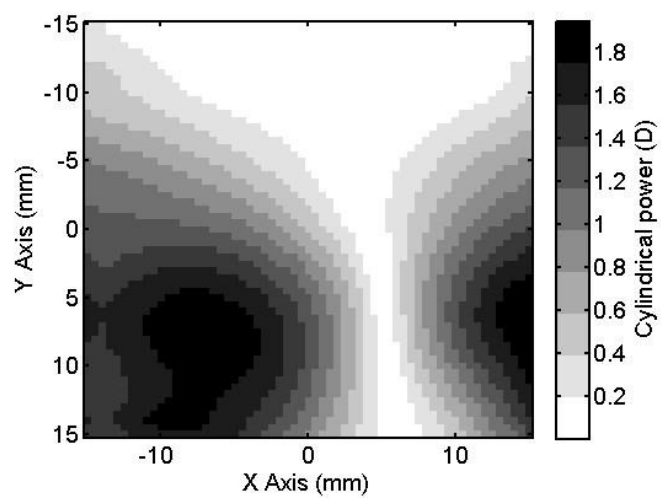

Figure 4a) Distribution of the spherical refractive power over a progressive surface, b) distribution of the cylindrical power for the same surface.

\section{CONCLUSIONS AND FUTURE WORK}

A CMM machine specifically suited for the measurement of "free-form" ophthalmic surfaces, particularly for progressive ones have been designed and assembled. The CMM employs an optical and a mechanical probe for measuring the surface. The optical probe will be used for the fast measurement of surfaces prone to suffer damage if they were measured using the mechanical one. The device is also formed by five motorized stages for the displacement of lens and positing of the sensor and also to change the orientation of the surface in order to extend the range of available curvatures that can be measured when the optical probe is used. All the components are assembled using custom made mechanical pieces designed by means of a CAD software and a custom made electronics have been developed to achieve the necessary degree of synchronization to perform fast measurements.

The goal of the profilometer is the fast and reliable measurement of ophthalmic lenses surfaces for a range of local curvatures ranging from 0 to $15 \mathrm{D}$ with an accuracy of $0.01 \mathrm{D}$ in local curvature. To achieve this goal it is necessary to measure the $\mathrm{X}, \mathrm{Y}$ and $\mathrm{Z}$ coordinates of the points of the surface with an accuracy of less than 1 micron. Moreover, due to the unavoidable systematic errors, particularly the squareness error, it is necessary to calibrate the device prior to measurement. This calibration is performed by the measurement of a perfect sphere using a least squares fitting algorithm to find the values of the parameters that account for the squareness error.

We have shown the results obtained after measuring a spherical and a progressive surface finding that the performance of the device is within specifications. However, these measurements have been carried out using the three axis configuration with mechanical probe as the software and algorithms necessary to orientate and measure with the optical probe lens surfaces presenting high slopes is still under development. When this work had been finished we will have a flexible device for the accurate measurement of "free-form" surfaces not restricted to ophthalmic surfaces.

\section{ACKNOWLEDGEMENTS}

The authors wish to thank the financial support of this work given by the Spanish Ministerio de Economía y Competitividad through the grant DPI2012-36103. 


\section{REFERENCES}

[1] Meister, D.J., Fischer, S.W., "Progress in the spectacle correction of presbyopia. Part 1: Design and development of progressive lenses," Clin. Exp. Optometry 91(3), 240-250 (2008).

[2] Meister, D.J., Fischer, S.W., "Progress in the spectacle correction of presbyopia. Part 2: Modern progressive lens technologies," Clin. Exp. Optometry 91(3), 251-264 (2008).

[3] Fabian Duerr, Youri Meuret, and Hugo Thienpont, "Potential benefits of free-form optics in on-axis imaging applications with high aspect ratio," Opt. Express 21, 31072-31081 (2013).

[4] W. T. Plummer, J. G. Baker, and J. Van Tassell, "Photographic optical systems with nonrotational aspheric surfaces," Appl. Opt. 38, 3572-3592 (1999).

[5] J. M. Infante Herrero, F. Muñoz, P. Benítez, J. C. Miñano, L. Wang, J. Vilaplana, G. Biot, and M. de La Fuente, "Novel fast catadioptric objective with wide field of view," Proc. SPIE 7787, 778704 (2010).

[6] Zheng Zhenrong, Hao Xiang, and Liu Xu, "Freeform surface lens for LED uniform illumination," Appl. Opt. 48, 6627-6634 (2009).

[7] Aleksandra Cvetkovic, Maikel Hernandez, Pablo Benítez, Juan C. Miñano, Joel Schwartz, Adam Plesniak, Russ Jones, David Whelan, "The free form XR photovoltaic concentrator: a high performance SMS3D design," Proc. SPIE 7043, 70430E (2008).

[8] JA Gómez-Pedrero, J Alonso, H Canabal, E Bernabeu, "A generalization of Prentice's law for lenses with arbitrary refracting surfaces," Ophthal. Physiol. Opt 18, 514-520 (1998).

[9] Heidenhain GmbH. Product MT2584 Brochure, <http://product.heidenhain.de/JPBC/image/MT_TIS.EN/hlrsystem/208945-2e length gauges.pdf $>$ (26 July 2015).

[10] Stil Optics S.A., web catalog, $<$ http://www.stilsa.com/catalog2/pdf/STILSA_Initial_CCS_CHR.pdf $>$ (26 July 2015)

[11] Arduino web site, <https://www.arduino.cc/> (26 July 2015).

[12] D. Rodriguez, J. Alonso, J.A. Quiroga, "Squareness error calibration of a CMM for quality control of ophthalmic lenses,” Int J Adv Manuf Technol 68, 487-493 (2013). 